\title{
Solar flare hard X-rays measured by spectrometer "IRIS": spectral and temporal characteristics
}

\author{
Yu.E. Charikov, P.B. Dmitriyev, I.V. Koudriavtsev, V.P. Lazutkov, \\ G.A. Matveev, M.I. Savchenko \& D.V. Skorodumov
}

Ioffe Physico-Technical Institute, Russian Academy of Sciences, Politekhnicheskaya str. 26, 194021 St.Petersburg, Russia e-mail: Yuri.Charikov@mail.ioffe.ru

\begin{abstract}
Sub-second time structure of hard X-rays observed during solar flares by spectrometer "IRIS" on a board of CORONAS satellite are discussed. The burst duration of $80 \mathrm{~ms}$ and FWHM about 30ms are recorded in powerful flare of August 20, 2002 year at 0828:16.77 UT. A confidence level of this peak is $6 \sigma$.
\end{abstract}

\section{Superfine time structure of solar flares}

The most direct evidence of magnetic reconnection processes in solar flares is a fast time structure of hard X-rays. Sub-second pulses detected in nonthermal hard X-rays are generally interpreted as bremsstrahlung from electrons accelerated in low corona (Kiplinger et al. 1983; Aschwanden 2001). "IRIS" spectrometer employs scintillation and proportional counters with large-sized windows. Flare precursors, micro- and nanoflares as well as fine time structures of hard X-rays can be measured. Scintillation counters record hard X-rays in 24-156 keV energy range in four energy channels with time resolution $10.49 \mathrm{~ms}$ (flare mode) (Kocharov et al. 2002. Figure 1 shows time profiles of hard X-rays (top panel) detected in solar flare occurred in August 20, 2002 year at 0824:50 UT. The flare is powerful, optical importance is 1B, GOES class is M34 and coordinates are S10 W38. Numerous spikes are separated clear at decline phase of the flare. Outstanding spike of this flare marked by arrow in top panel observed at 08:28:16.77 UT (see low panel). Count rate registered over $24 \mathrm{keV}$ have been integrated. A confidence level of this peak is $6 \sigma$, time profile includes 8 time points followed each other in $10.49 \mathrm{~ms}$ interval (low panel). These facts witness the non-accidental physical nature of this pulse. Time profile is asymmetrical: rise phase lasts $20 \mathrm{~ms}$, sharp peak replaced by decline phase with $60 \mathrm{~ms}$ decay. The burst duration is $80 \mathrm{~ms}$ and FWHM about $30 \mathrm{~ms}$. It should be knouted the same pulse structure did not observe at the rise and peak phases of the flare. Another case when spiky structure observed at different phases is shown in figure 2. Time profile of solar flare 26.08.2002 consist of some pulses with width of $>30 \mathrm{~ms}$. At the rise phase of the flare two pulses have a confidence level equalled to $3 \sigma$. At the peak stage the one spike has a $4 \sigma$ confidence level and confidence level of another spike is $3 \sigma$. The same situation repeated at decline phase. Full widths at FWHM of these spikes are 20-30ms. The grows time of the tearing mode and coalescence time for coronal plasma and magnetic field are 0.2-0.4s comparable with spike duration (Aschwanden 2001) 

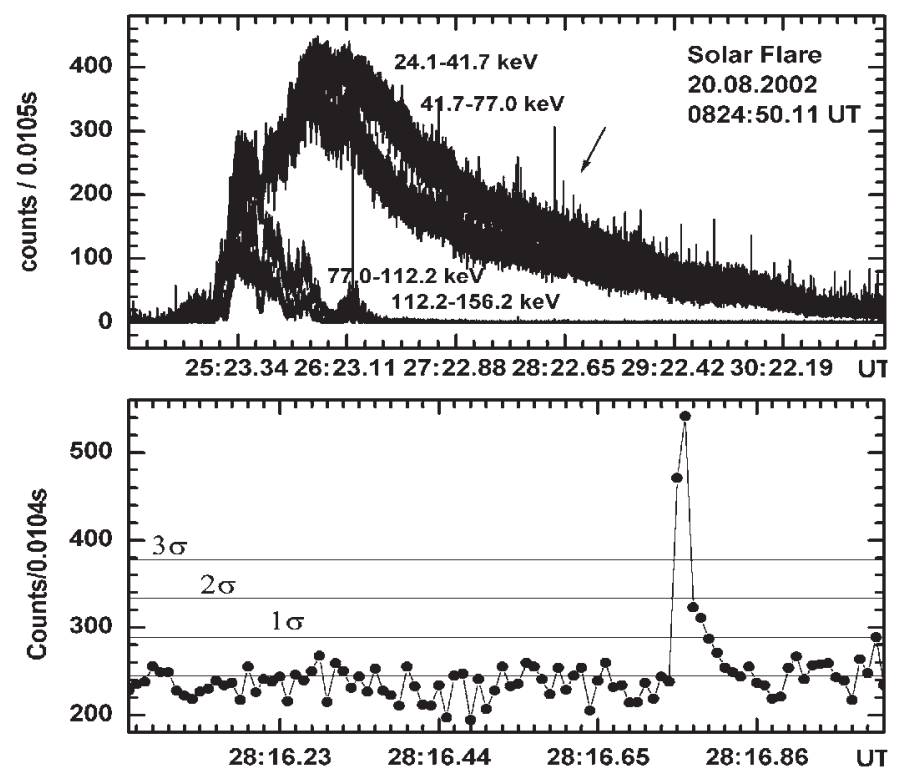

Figure 1. Hard X-ray time profiles of 20.08.2002 solar flare (top) and sub-second spike. Horisontal lines represent a confidence levels of $1 \sigma$ to $3 \sigma$.
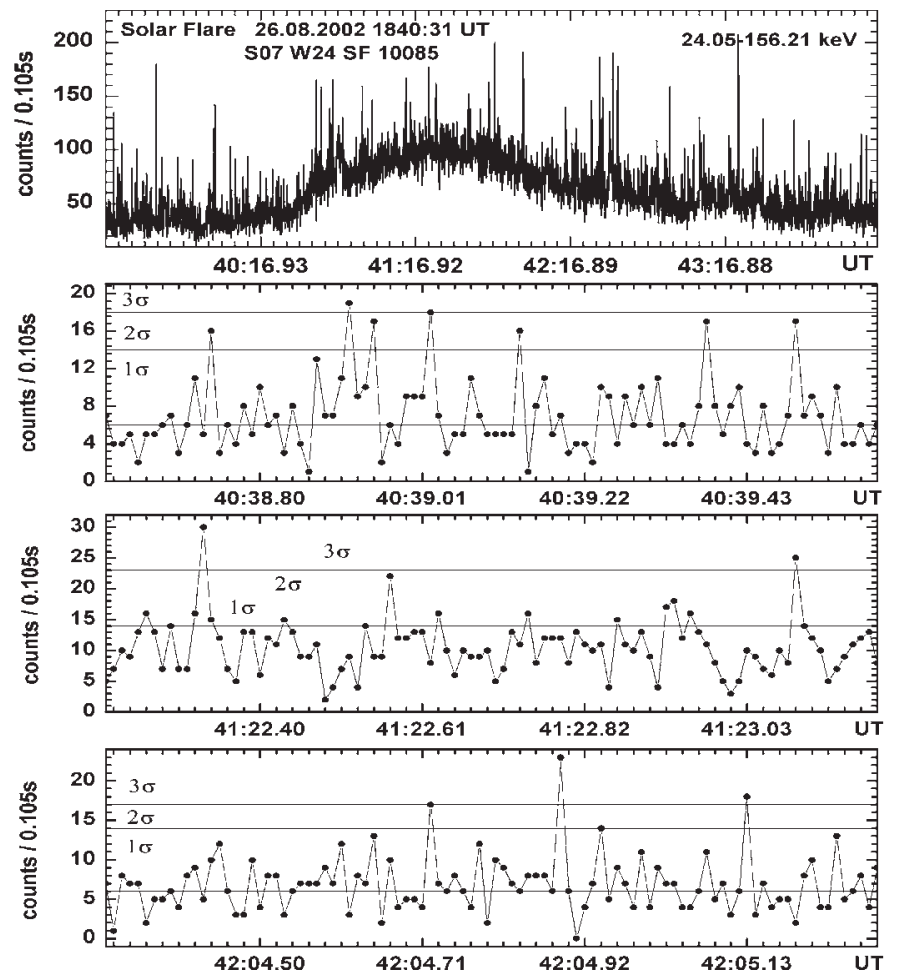

Figure 2. Hard X-ray time profiles of 26.08.2002 solar flare (top) and sub-second spikes at different phases of the flare. Horisontal lines represent a confidence levels of $1 \sigma$ to $3 \sigma$. 


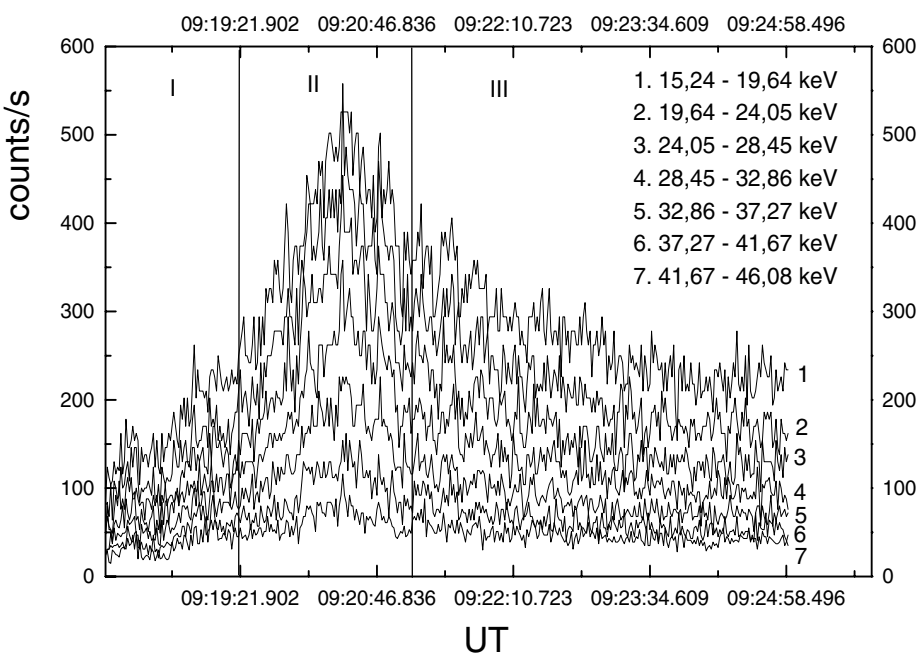

Figure 3. Hard X-ray time profiles of solar flare 26.07.02.

\section{Time-dependent hard X-ray energy spectra in solar flare 26.07.02 and 19.12.01.}

In the thick target model, electrons lose their energy and produce a photon in bremsstrahlung. The hard X-ray energy spectrum determines the spectrum of fast electrons accelerated in solar flares. "IRIS" scintillation counters measure a photons in 32 energy channels from $15.24 \mathrm{keV}$ to $156.2 \mathrm{keV}$. Time resolution of this registration is 1 second. The analysis of hard X-rays detected in this energy passband allow us to calculate time dependent energy spectra with high accuracy. We analyze hard X-rays of solar flare occurred 26.07.2002 year at 9:20 UT. Optical importance of the flare is SF. Hard X-ray time profiles for different energies are shown in Fig. 3. Individual pulses with width of some seconds compose time profiles.

Time-dependent energy spectra have been calculated at rise (I), peak(II) and decline(III) flare phases. Fig.4 shows a spectra at different phasesfor different times followed each other via 1 second. At rise phase and peak phase a spectra can not fit to power law with a unique slope, the higher energy the steeper spectra (fig.4a). At decline phase a spectra obey a power law (fig.4b) with average slope $\gamma=-1.64$ (straight line).

The analyses of hard X-rays for another events show the same time dependence of a spectra. Fig.5 shows a spectra of flare 19.12.01 at the moment of peak phase and the results of hard X-ray energy spectra modeling. In the thick target model, we calculate the bremsstrahlung of the fast electrons, initial velocity distribution function of which is a $\mathrm{H}(\mathrm{v}$-vmax $)$ - Heaviside unit function. The high limit of electron energy is varied. Evolution of this function have been calculated take into account the Coulomb collisions. The platodistribution function with sharp decline can be resulted of nonlinear relaxation electron beam due to the generation and collisions electrons with plasma waves and nonlinear scattering of Langmuire plasmons on thermal plasma particals (Kudryavtsev \& Charikov 1999).

\section{References}

Kiplinger, A.L., Dennis, B.R., Frost, K.J. Orwig, L.E. 1983 Astrophys. J. 229, L99. 

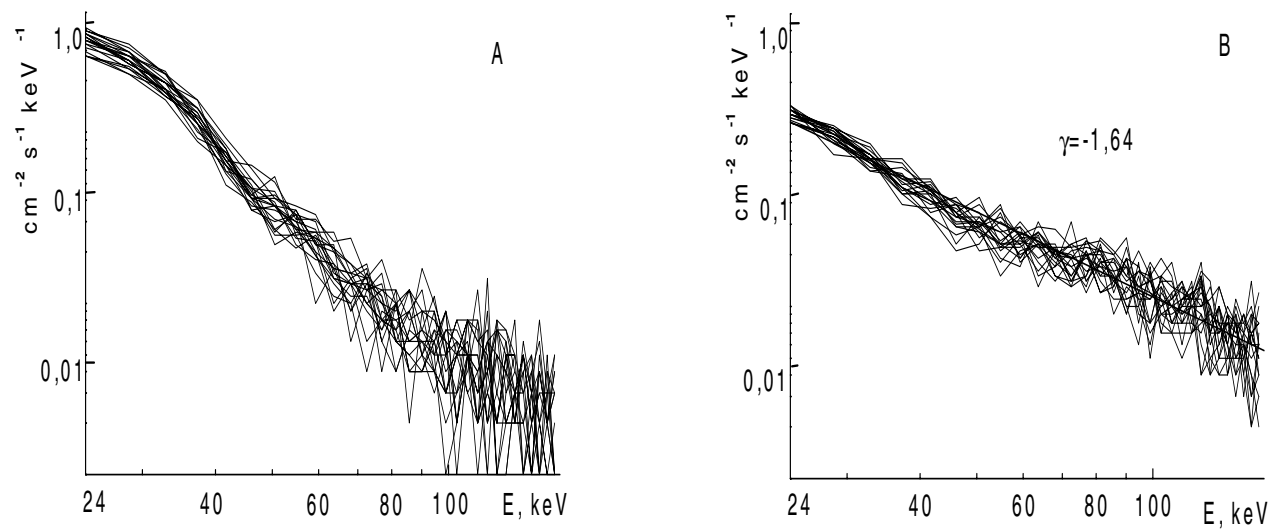

Figure 4. Hard X-ray spectra of flare 26.07.02 on the peak (a) and decline (b) phases.

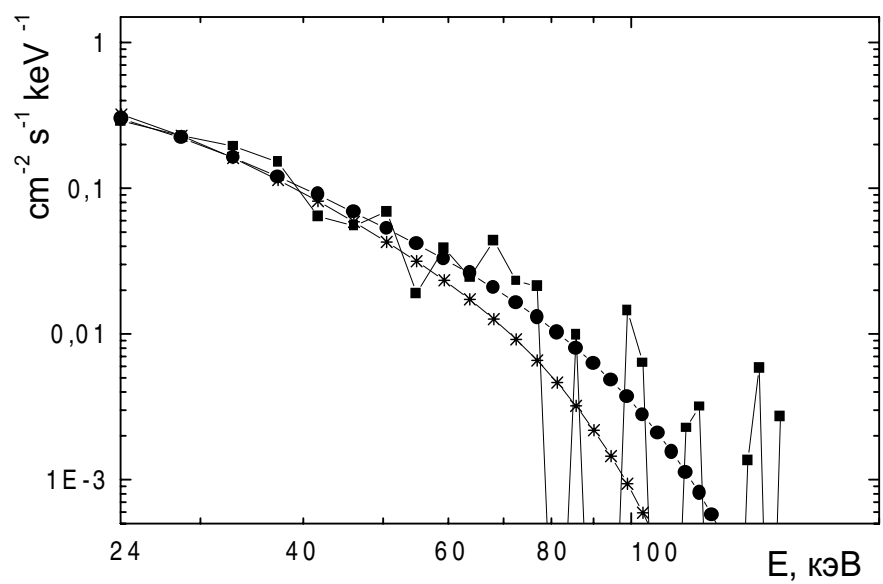

Figure 5. - IRIS hard X-ray spectra of 19.12.01in the peak phase; $*$, $\bullet$ - are modeling spectra $E \max =110 \mathrm{keV}, E \max =140 \mathrm{keV}$.

Aschwanden, M.J. 2001 In "Particle acceleration and kinematics in solar flares" Kluwer Ac.Publ., p. 227

Kocharov,G,E., Lazutkov,V.P., Matveev,G.A.,Nitsora, Ju.N., Savchenko,M.I., Skorodumov,

D.V., Oraevskii,V.N., Stepanov,A.I. \& Lisin 2002 In "Active Processes at the Sun and Stars", Proc. Inter.Conf. St.-Petersburg, 1-6 July,2002, 314.

Koudriavtsev,I.V. \& Charikov,Ju.E. 1999 Astronomy Reports 43(6), pp. 400-405 